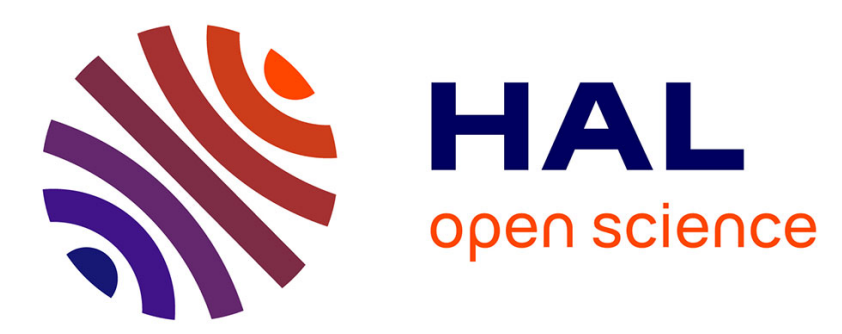

\title{
Thermal conductivity and thermoelectric power of $\mathrm{UBe} 13$
}

\author{
D. Jaccard, J. Flouquet, Z. Fisk, J.L. Smith, H.R. Ott
}

\section{To cite this version:}

D. Jaccard, J. Flouquet, Z. Fisk, J.L. Smith, H.R. Ott. Thermal conductivity and thermoelectric power of UBe13. Journal de Physique Lettres, 1985, 46 (17), pp.811-817. 10.1051/jphyslet:019850046017081100 . jpa-00232903

\section{HAL Id: jpa-00232903 https://hal.science/jpa-00232903}

Submitted on 1 Jan 1985

HAL is a multi-disciplinary open access archive for the deposit and dissemination of scientific research documents, whether they are published or not. The documents may come from teaching and research institutions in France or abroad, or from public or private research centers.
L'archive ouverte pluridisciplinaire HAL, est destinée au dépôt et à la diffusion de documents scientifiques de niveau recherche, publiés ou non, émanant des établissements d'enseignement et de recherche français ou étrangers, des laboratoires publics ou privés. 
Classification

Physics Abstracts

$72.15 \mathrm{E}-72.15 \mathrm{~J}-74.30 \mathrm{E}$

\title{
Thermal conductivity and thermoelectric power of $\mathbf{U B e}_{13}$
}

\author{
D. Jaccard, J. Flouquet
}

Centre de Recherches sur les Très Basses Températures, CNRS, BP 166 X, 38042 Grenoble Cedex, France

\author{
Z. Fisk, J. L. Smith \\ Materials Science and Technology Division, Los Alamos National Laboratory, Los Alamos, \\ NM 87545, U.S.A.
}

and H. R. Ott

Laboratorium für Festkörperphysik, Eidgenössische Technische Hochschule, Hönggerberg, CH-8093 Zürich, Switzerland

(Reçu le 8 avril 1985, accepté sous forme définitive le 8 juillet 1985)

\begin{abstract}
Résumé. - Des mesures de résistivité, pouvoir thermoélectrique et de conductivité thermique du composé à fermions lourds $\mathrm{UBe}_{13}$ ainsi que leurs relations avec le diagramme de phase températurechamp critique sont présentées. A basse température $(T<1 \mathrm{~K})$, le pouvoir thermoélectrique de la phase normale est gigantesque. La conductivité thermique suit une loi en $T^{2}$ dans la phase supraconductrice sans contribution linéaire en $T$ contrairement à ce qui est observé dans $\mathrm{UPt}_{3}$.

Abstract. - Measurements of the resistivity, thermoelectric power and thermal conductivity of the heavy fermion compound $\mathrm{UBe}_{13}$ and their relations to the temperature-upper critical field phase diagram are reported. At low temperature $(T<1 \mathrm{~K})$, enormous thermoelectric powers are detected in the normal phase. The thermal conductivity obeys a $T^{2}$ law in the superconducting phase with no supplementary $T$ contribution which is not observed in the other heavy fermion compound $\mathrm{UPt}_{3}$.
\end{abstract}

\section{Introduction.}

The discovery of superconductivity in $\mathrm{UBe}_{13}$ at $T_{\mathrm{c}} \sim 0.9 \mathrm{~K}$ [1] has revived the debate on the origin of the superconductivity in heavy fermion compounds first found in $\mathrm{CeCu}_{2} \mathrm{Si}_{2}$ [2]. The heavy fermion character of $\mathrm{UBe}_{13}$ is proved by the large value of the ratio of the specific heat $C$ to the temperature $T$ near $T_{\mathrm{c}}: C / T \sim 1 \mathrm{~J} / \mathrm{mole} / \mathrm{K}^{2}$ [3]. One of the peculiarities of $\mathrm{UBe}_{13}$ is that the electron mean free path is extremely short near $T_{\mathrm{c}}$. At $1 \mathrm{~K}$, the electrical resistivity $(\rho \sim 190 \mu \Omega \mathrm{cm})$ [1-4] is almost that estimated for the unitary limit; no sign of Fermi liquid behaviour $\left(\rho \sim T^{2}\right)$ is detected. By contrast with liquid ${ }^{3} \mathrm{He}$ for which the superfluid transition $\left(T_{\mathrm{c}} \sim 2 \mathrm{mK}\right)$ is far below the Fermi temperature $T_{F}$ [5], the definition of $T_{F}$ is not obvious. 
To clarify the specific case of $\mathrm{UBe}_{13}$, a study of transport properties will be reported. Measurements of the thermoelectric power $Q$ provide a sensitive method for mapping the density of electronic states and simultaneous measurements of the thermal conductivity are performed in both normal (N) and superconducting phase (S).

\section{Experimental set up.}

The single crystalline $\mathrm{UBe}_{13}$ sample has been prepared as described in reference [1]. The sample was a parallelepiped with cross sectional area $0.63 \mathrm{~mm}^{2}$ and length $1.5 \mathrm{~mm}$. The temperature gradient $\nabla T$ is measured by two $68 \Omega$ Matshushita resistors soldered with indium at each extremity. A four lead measurement gives the heater power. The thermoelectric voltage is detected by a conventional nanovoltmeter manufactured by Tekelec with a sensitivity better than $2 \mathrm{nV}$.

The experiments are done with magnetic fields $(H)$ up to $75 \mathrm{kOe}$ in a dilution refrigerator cryostat. An auxiliary Matshushita resistor is located in the compensated region of the superconducting magnet for correcting the thermometers for magnetoresistivity. The lowest applied field $H$, used to quench the superconductivity of indium, is $1 \mathrm{kOe}$. The magnetic field, the electric current and the thermal gradient were applied along the length of the sample i.e. parallel to a primitive vector of the cubic Bravais lattice.

\section{Results.}

3.1 Resistivity $(\rho)$. - As previously reported [1], a maximum of paramagnetic scattering $\rho_{\mathrm{M}} \sim 220 \mu \Omega \mathrm{cm}$ is observed at $T_{\mathrm{M}}^{\rho} \sim 2.5 \mathrm{~K}$. By resistivity measurements, the superconducting transitions $T_{\mathrm{c}}$ at 0 and $75 \mathrm{kOe}$ are respectively $854 \mathrm{mK}$ and $220 \mathrm{mK}$ with widths (defined by $10 \%$ and $90 \%$ changes in $\rho$ ) equal to $12.5 \mathrm{mK}$ and $58 \mathrm{mK}$. The upper critical field $H_{\mathrm{c}_{2}}(T)$ derived from resistivity confirms the published phase diagrams with an enormous initial slope $\left.\frac{\partial H_{\mathrm{c}_{2}}}{\partial T}\right|_{T_{\mathrm{c}}}>$ - $270 \mathrm{kOe} / \mathrm{K}$ (regime I for $H<15 \mathrm{kOe}$ ) followed by a quasi-linear temperature dependence down to $0 \mathrm{~K}$ with a slope $\left.\frac{\partial H_{\mathrm{c}_{2}}}{\partial T}\right|_{T \rightarrow 0} \sim-98 \mathrm{kOe} / \mathrm{K}$ (regime II for $H>15 \mathrm{kOe}$ ).

3.2 THERMOELECTRIC POWER $(Q)$. - Figure 1 shows the temperature variation of $Q$ for different applied fields. In low fields $(H \sim 1 \mathrm{kOe})$, the striking features are the enormous value of $Q$ $(\sim-24 \mu \mathrm{V} / \mathrm{K})$ at $T_{\mathrm{c}}^{+}$just above $T_{\mathrm{c}}$ and the increase of its amplitude on cooling. The proportionality of $Q$ to $T$ that is usual for metal at low $T$ is not found. If the normal state could persist at $0 \mathrm{~K}, Q$ would pass through a minimum below $0.8 \mathrm{~K}$. Qualitatively, this behaviour implies the occurrence of a structure in the density of states with a characteristic energy $\lesssim 1 \mathrm{~K}$.

Another interesting result is that, in the regime I of the phase diagram, $\frac{\partial Q}{\partial T}>0$ while in II, $\frac{\partial Q}{\partial T}<0$. Furthermore, the field dependence of $Q\left(T_{\mathrm{c}}^{+}\right)$is quasi-linear in $H$ with an extrapolation to zero at $H=78 \mathrm{kOe} \lesssim H_{\mathrm{c}_{2}}(0)$ (insert b); the superconductivity is destroyed when $Q(T)$ becomes positive for $H>H_{\mathrm{c}_{2}}(0)$. In $\mathrm{CeAl}_{3}$ [6], positive thermopower has been observed at low temperatures just when $\rho$ follows a $T^{2}$ dependence.

3. 3 Thermal CONDUCTIVITY $(\kappa)$. - Figure 2 presents the thermal conductivity at two different fields $H=1 \mathrm{kOe}$ and $75 \mathrm{kOe}$. For $H=75 \mathrm{kOe}, \kappa$ follows a linear temperature dependence in the normal phase well below $T_{\mathrm{M}}^{\rho}$. 


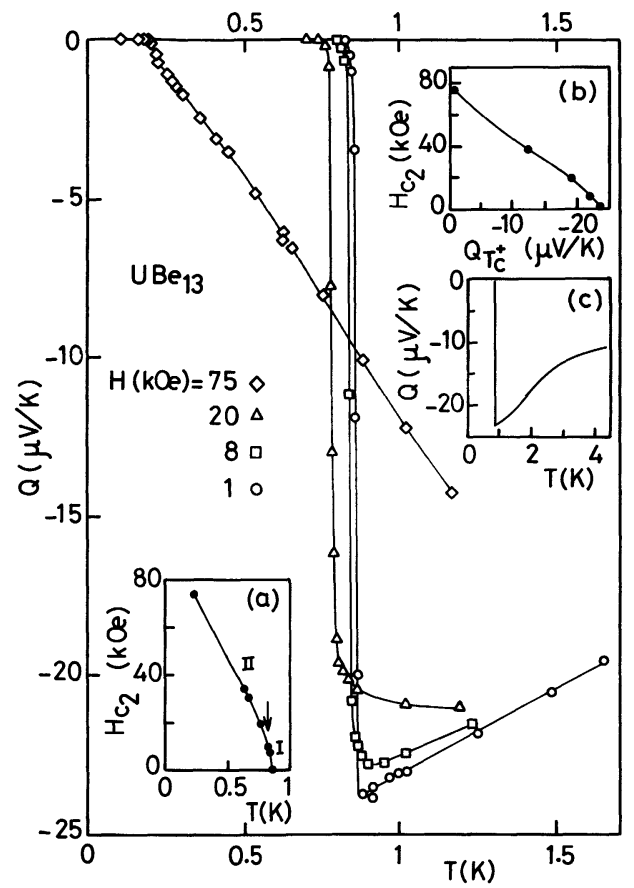

Fig. 1. - Thermoelectric power $Q$ of $\mathrm{UBe}_{13}$ as a function of $T$ for $H=1,8,20$ and $75 \mathrm{kOe}$. Insert (a) is the $H_{\mathrm{c}_{2}}(T)$ phase diagram obtained by resistivity measurements, insert (b) shows a phase diagram drawn with $Q\left(T_{\mathrm{c}}^{+}\right)$as the variable and insert (c) is for $H=1 \mathrm{kOe}, Q(T)$ up to $4.2 \mathrm{~K}$.

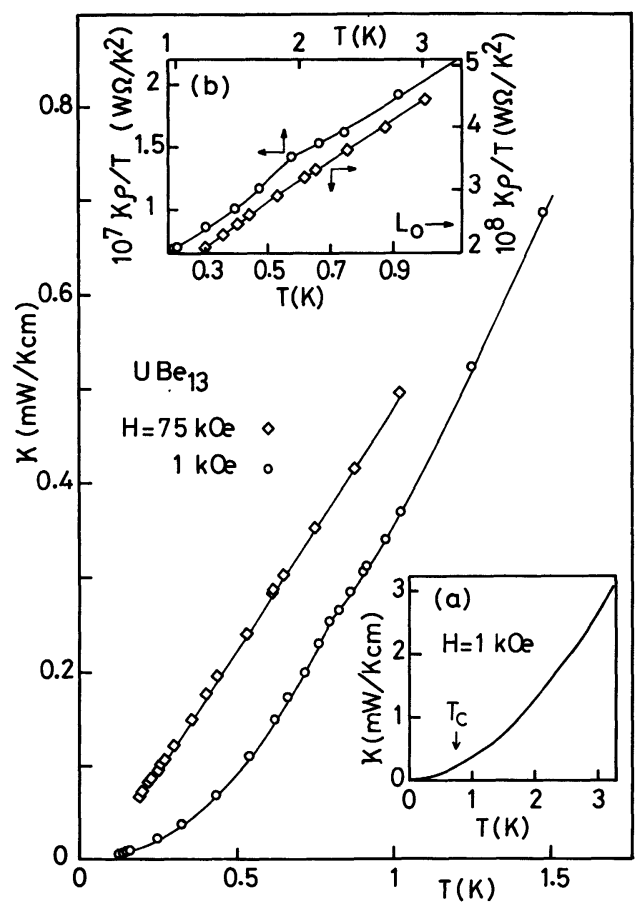

Fig. 2. - Thermal conductivity $\kappa$ at $H=1$ and $75 \mathrm{kOe}$. Insert (a) shows the variation of $\kappa$ at $H=1 \mathrm{kOe}$ up to $3.5 \mathrm{~K}$, and insert (b) the quasilinear $T$ dependence of the « Lorentz number » $\kappa \rho / T$. 


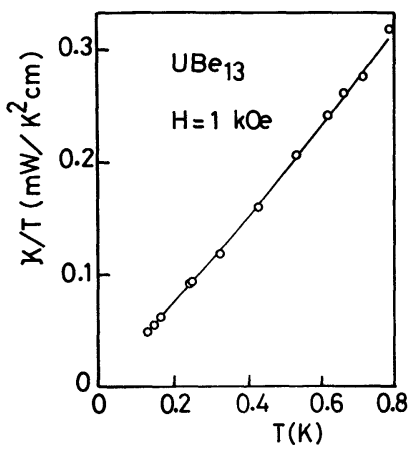

Fig. 3. - In the superconducting phase of $\mathrm{UBe}_{13}, \kappa / T$ as a function of $T$.

At $1 \mathrm{kOe}$, a kink in $\kappa$ is observed at $0.79 \mathrm{~K}$ i.e. $65 \pm 5 \mathrm{mK}$ below $T_{\mathrm{c}}(\rho)$. This discrepancy is probably due to the inhomogeneity of the sample. In the superconducting state almost up to $T_{\mathrm{c}}$, $\kappa_{\mathrm{s}}$ has a $T^{2}$ dependence as shown in figure 3 . The contribution of the phonons is difficult to establish with certainty. However, a high Debye temperature $\left(\theta_{\mathbf{D}} \sim 840 \mathrm{~K}\right)$ [7] and the imperfections of the lattice limiting the phonon mean free path favour a weak contribution below $1 \mathrm{~K}$. Above $1 \mathrm{~K}$, an experimental proof of the importance of the electronic contribution is already given by the fact that the maximum in $\rho$ at $T_{\mathrm{M}}^{\rho} \sim 2.5 \mathrm{~K}$ is connected with a change in the thermal conductivity at $T=2.2 \mathrm{~K} \sim T_{\mathrm{M}}^{\rho}$.

The measurements of $\kappa$ and $\rho$ permit a comparison of their product with the well-known Wiedemann-Franz law observed for elastic scattering processes of fermions :

$$
\rho_{0} \kappa_{0}=L_{0} T
$$

with $L_{0} \sim 2.45 \times 10^{-8} \mathrm{~W} \Omega \mathrm{K}^{-2}$. As shown in the insert $\mathrm{b}$ of figure $2, \kappa \rho / T$ shows quasi-linear temperature dependences in low field $(H \sim 1 \mathrm{kOe})$ and high field $(H=75 \mathrm{kOe}):$ at $1 \mathrm{~K}$, respective values are $6.8 \times 10^{-8} \mathrm{~W} \Omega \mathrm{K}^{-2}$ and $4.5 \times 10^{-8} \mathrm{~W} \Omega \mathrm{K}^{-2}$ for $H=1 \mathrm{kOe}$ and $75 \mathrm{kOe}$.

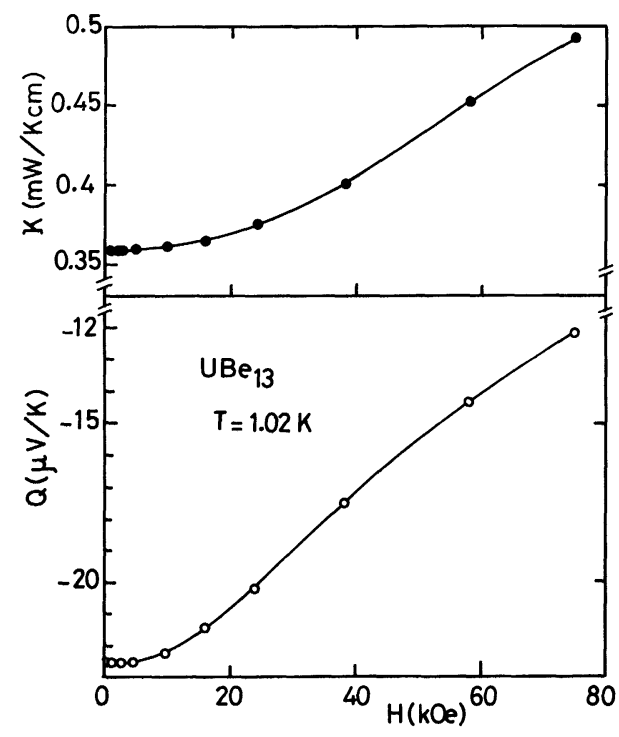

Fig. 4. - Field dependence of $\kappa$ and $Q$ at $1.02 \mathrm{~K}$. 
This unusual behaviour points out that, in $\mathrm{UBe}_{13}$, the carriers of electricity and heat may differ or that, as it has been emphasized in magnetoresistivity experiments [8], below $100 \mathrm{kOe}$, there is a strong interplay between Fermi liquid and scattering disorder phenomena; when, at low temperatures, the $\rho$ data is analysed with residual plus $T^{2}$ terms, different field dependence must be given to the two contributions [8].

Figures 4 and 5 reproduce the field dependence of $\kappa$ and $Q$ at $1.02 \mathrm{~K}$ and at $655 \mathrm{mK}$. In the normal phase, the low field variation of $\kappa$ and $Q$ is weak; a field sweep at $655 \mathrm{mK}$ shows a quasi quadratic dependence in $H$ below $H_{\mathrm{c}_{2}}$ and a linear variation above $H_{\mathrm{c}_{2}}$.

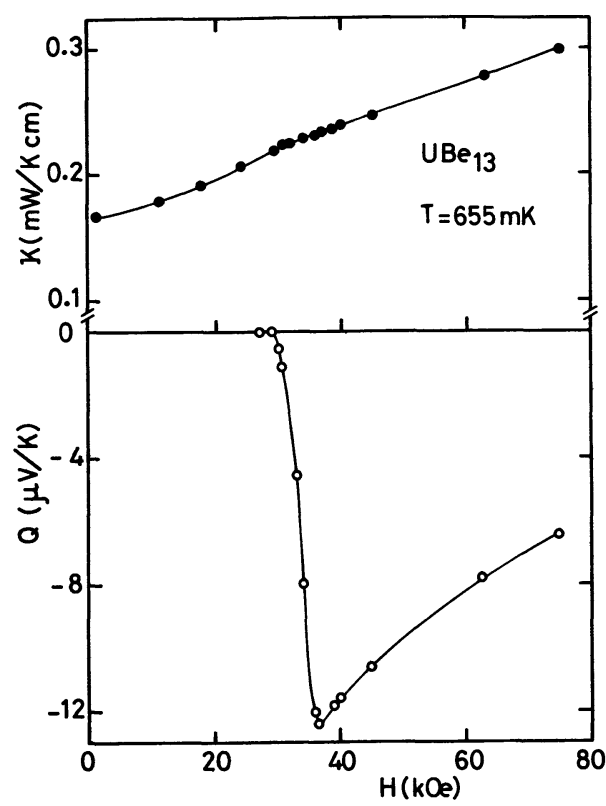

Fig. 5. - Field dependence of $\kappa$ and $Q$ at $655 \mathrm{mK}$.

\section{Discussion.}

The quasi-linear temperature dependence of the Lorentz number $\kappa \rho / T$, the possible occurrence at low field of a minimum in $Q$ below $T_{\mathrm{c}}$ and the huge value of the resistivity indicate that, in $\mathrm{UBe}_{13}$, the Fermi liquid regime is far from being achieved at $T_{\mathrm{c}}$. In low fields, clearly a large disorder occurs; in regime $I$, the scattering disorder may reinforce the superconducting state as suggested for weakly localized, nearly magnetic systems [9].

In the superconducting state, the $T^{2}$ dependence of $\kappa$ appears to be another demonstration of unusual superconducting pairing $[10,11]$. Such a variation has been predicted for a polarlike state in odd pairing $[12,13]$. However group-theoretical arguments seem to reject such a state $[14,15]$, although it may be allowed in the presence of spin anisotropy in the pairing interactions [13].

In many aspects, $\mathrm{UBe}_{13}$ is an unusual heavy fermion compound. The exotic heavy fermion properties are usually observed for compounds with a strong axial symmetry $\left(\mathrm{CeAl}_{3}, \mathrm{CeCu}_{2} \mathrm{Si}_{2}\right.$, $\mathrm{UPt}_{3}$ ) and not for cubic materials like $\mathrm{UBe}_{13}$. An interesting idea is that the hybridization matrix elements are extremely anisotropic and that the cubic symmetry can be spontaneously broken [7]. Thermal conductivity experiments must be performed below $100 \mathrm{mK}$ to test the picture of strong anisotropy of the energy gap with BCS singlet pairing [7]. 
It is worthwhile to mention that the three well-known heavy fermion superconductors have quite different magnitudes of the resistivity at $T_{c}^{+}$(or of their extrapolated value $\rho(0)$ at $T \rightarrow 0$ ), of the thermoelectric power at $T_{\mathrm{c}}^{+}$and of the ratio of $T_{\mathrm{c}}$ by $T_{\mathrm{M}}^{\rho}$ when a clear maximum can be observed in resistivity data (Table I). There is no doubt [17] that $\mathrm{UPt}_{3}$ is a clean superconductor with an electronic mean free path $(l \sim 3000 \AA)$ higher than the coherence length $\left(\phi_{0} \sim 100 \AA\right)$ whereas crude estimates of $l \leqslant 30 \AA$ and $\phi_{0} \sim 50 \AA$ in $\mathrm{UBe}_{13}$ classify it as a dirty superconductor [4].

Table I. - Resistivity $\rho\left(T_{\mathrm{c}}^{+}\right)$and thermoelectric power $Q\left(T_{\mathrm{c}}^{+}\right)$just above $T_{\mathrm{c}}$, ratio of the superconducting transition $T_{\mathrm{c}}$ to the temperature $T_{\mathrm{M}}^{\rho}$ of the maximum in resistivity and estimated residual resistivity.

\begin{tabular}{l|c|c|c|c|c} 
& $\begin{array}{c}\rho\left(T_{\mathrm{c}}^{+}\right) \\
\mu \Omega \mathrm{cm}\end{array}$ & $\begin{array}{c}Q\left(T_{\mathrm{c}}^{+}\right) \\
\mu \mathrm{V} / \mathrm{K}\end{array}$ & $T_{\mathrm{c}} / T_{\mathrm{M}}^{\rho}$ & $\begin{array}{c}\rho(0) \\
\mu \Omega \mathrm{cm}\end{array}$ & $\operatorname{Ref.}$ \\
\hline $\mathrm{UBe}_{13}$ & $\sim 180$ & -24 & 0.36 & 40 & {$[1,4,8]$} \\
$\mathrm{CeCu}_{2} \mathrm{Si}_{2}$ & $\sim 15$ & -3 & 0.03 & 5 & {$[16]$} \\
$\mathrm{UPt}_{3}$ & $\sim 1.5$ & +0.5 & & $<0.6$ & {$[17]$}
\end{tabular}

At the difference of this diversity, the thermal conductivity of the three heavy fermion compounds follows

$$
\kappa_{\mathrm{s}}=\alpha T^{2}+\beta T \quad \text { for } \quad T<T_{\mathrm{c}} .
$$

The parameters $\alpha$ and $\beta$ are reported in table II. For $\mathrm{UBe}_{13}$, a quadratic term alone is observed. Renormalization by the thermal conductivity at $T_{\mathrm{c}}$ gives a dimensionless parameter $\alpha T_{\mathrm{c}}^{2} / \kappa\left(T_{\mathrm{c}}\right)$ almost equal to 1 for $\mathrm{UBe}_{13}$ and $\mathrm{CeCu}_{2} \mathrm{Si}_{2}$. This suggests a similarity between both compounds which has already been observed in the temperature dependence of their specific heats [18] (a $T$ exponent upper than two has been found for $\mathrm{UBe}_{13}$ and $\mathrm{CeCu}_{2} \mathrm{Si}_{2}$ [18] while $T$ plus $T^{2}$ terms have been reported for $\left.\mathrm{UPt}_{3}[19,20]\right)$. In our opinion, the linear temperature term in $\kappa$

Table II. - Coefficients $\alpha$ and $\beta$ of the $T^{2}$ and $T$ term of the thermal conductivity in the superconduction state.

\begin{tabular}{l|c|c|c|c|c|c} 
& $\begin{array}{c}\alpha \\
\mathrm{mW} / \mathrm{K}^{3} \mathrm{~cm}\end{array}$ & $\begin{array}{c}\beta \\
\mathrm{mW} / \mathrm{K}^{2} \mathrm{~cm}\end{array}$ & $\begin{array}{c}\kappa\left(T_{\mathrm{c}}\right) \\
\mathrm{mW} / \mathrm{K} \mathrm{cm}\end{array}$ & $\frac{\alpha T_{\mathrm{c}}^{2}}{\kappa\left(T_{\mathrm{c}}\right)}$ & $\begin{array}{c}T_{\mathrm{c}} \\
\mathrm{mK}\end{array}$ & Ref. \\
\hline $\mathrm{UBe}_{13}$ & 0.38 & 0 & 0.25 & 1.08 & 854 & \\
$\mathrm{CeCu}_{2} \mathrm{Si}_{2}$ & 2.8 & 0.7 & 0.8 & 1.11 & 560 & \\
$\mathrm{UPt}_{3}$ & 19.5 & 0.55 & 3.1 & 1.70 & 520 & {$[17]$}
\end{tabular}

observed in $\mathrm{CeCu}_{2} \mathrm{Si}_{2}$ is a parasitic effect coming from the difficulty of producing a homogeneous sample of the required size; the $\mathrm{CeCu}_{2} \mathrm{Si}_{2}$ reported results are on a dense unannealed sample with a low content of microcracks; the large broadening of the superconducting transition measured by susceptibility [16] suggests that a larger part of the sample stays in a normal state and that the $T$ term has then a parasitic origin.

However, the observation in $\mathrm{UPt}_{3}$ of a $T$ term in the specific heat well below $T_{\mathrm{c}}$ (at $T=146 \mathrm{mK} \sim 0.28 T_{\mathrm{c}}$ ) [19] may suggest that here a normal component could exist in the 
superconducting phase. The interesting conclusion would be that the $\beta T$ contribution in $\kappa$ is an intrinsic effect due to the motion of the normal component. If the gap vanishes along lines on the Fermi surface, only $T^{2}$ contributions have been predicted [12]. The intrinsic character of the $T$ terms in $\mathrm{UPt}_{3}$ is still an open experimental question.

\section{Conclusion.}

Extensive measurements of transport properties of $\mathrm{UBe}_{13}$ show clearly the complexity of this unusual system. A simple result is the $T^{2}$ dependence of its thermal conductivity. Important differences exist between the two uranium superconductors $\mathrm{UBe}_{13}$ and $\mathrm{UPt}_{3}$. The possible occurrence of normal component below $T_{\mathrm{c}}$ in $\mathrm{UPt}_{3}$ is underlined.

\section{Acknowledgments.}

The authors thank particularly Dr. N. Phillips, Dr. S. von Molnar and Dr. C. M. Varma for their encouragements and stimulating discussions. They are indebted to Dr. H. F. Braun and P. Lejay for preparing the $\mathrm{CeCu}_{2} \mathrm{Si}_{2}$ and $\mathrm{UPt}_{3}$ samples. Work at Los Alamos was performed under the auspices of the U.S. Department of Energy.

\section{References}

[1] Ott, H. R., Rudigier, H., Fisk, Z. and Smith, J. L., Phys. Rev. Lett. 50 (1983) 1595.

[2] Steglich, F., Aarts, J., Bredl, C. D., Lieke, W., Meschede, D., Franz, W. and Schäfer H., Phys. Rev. Lett. 43 (1979) 1892.

[3] Ott, H. R., Rudigier, H., Rice, T. M., Ueda, K., Fisk, Z. and Smith, J. L., Phys. Rev. Lett. 52 (1984) 1915.

[4] Maple, M. B., Chen, J. W., Lambert, S. E., Fisk, Z., Smith, J. L. and Ott, H. R., Phys. Rev. Lett. 54 (1985) 477.

[5] Osheroff, D. D., Richardson, R. C. and Lee, D. M., Phys. Rev. Lett. 28 (1972) 885.

[6] JACCARD, D., Flouquet, J., J. Magn. Magn. Mater. 47-48 (1985) 45.

[7] Overhauser, A. W. and Appel, J., Phys. Rev. B 31 (1985) 193.

[8] Remenyi, G., Briggs, A., Jaccard, D., Flouguet, J., Fisk, Z., Smith, J. L., to be published.

[9] Beal-Monod, M. T., Ebisawa, H. and Fukuyama, H., Phys. Rev. B 30 (1984) 1563.

[10] Bishop, D. J., Varma, C. M., BatlogG, B., Bucher, E., Fisk, Z. and Smith, J. L., Phys. Rev. Lett. 53 (1984) 1009.

[11] Maclaughlin, D. E., Tien, C., Clark, W. G., Glover, K., Lan, M. D., Fisk, Z., Smith, J. L. and OtT, H. R., Phys. Rev. Lett. 53 (1984) 1833.

[12] Varma, C. M., J. Appl. Phys. 57 (1985) 3065.

[13] Varma, C. M., to be published in Comments in Solid State Phys.

[14] Volovik, G. E. and Gorkov, L. P., Zh. Eksp. Teor. Fiz., Pisma Red (to be published).

[15] Anderson, P. W., Phys. Rev. B 30 (1984) 1549.

[16] Tholence, J. L., Haen, P., JacCard, D., Lejay, P., Flouquet, J. and Braun, H. F., to be published in J. Appl. Phys. Proceedings of MMM (1984).

[17] Jaccard, D., Flouquet, J., Lejay, P. and Tholence, J. L., J. Appl. Phys. 57 (1985) 3082.

[18] Stewart, G. R., Rev. Mod. Phys. 56 (1984) 755.

[19] Sulpice, A., Gandit, P., Chaussy, J., Flouquet, J., Jaccard, D. and Lejay, P., to be published.

[20] Steglich, F., Rauschswalbe, U., Gottwick, U., Mayer, H. M., Sparn, G., Grewe, N. and Franse, J. J. M., J. Appl. Phys. 57 (1985) 3054. 